\title{
Analysis and Preliminary Results for the Cosmic Ray Electron Spectrum from CALET
}

\author{
Yoichi Asaoka* for the CALET Collaboration \\ Research Institute for Science and Engineering, Waseda University, 3-4-1 Okubo, Shinjuku, \\ Tokyo 169-8555, Japan, and \\ JEM Mission Operations and Integration Center, Human Spaceflight Technology Directorate, \\ Japan Aerospace Exploration Agency, 2-1-1 Sengen, Tsukuba, Ibaraki 305-8505, Japan \\ E-mail: yoichi.asaoka@aoni.waseda.jp
}

CALET (CALorimetric Electron Telescope) mission was launched to the International Space Station (ISS) in August 2015 and has been accumulating scientific data since October 2015. The CALET detector, consisting of a charge detector (CHD), imaging calorimeter (IMC) and total absorption calorimeter (TASC), features a very thick calorimeter of 30 radiation-lengths. Due to its high energy resolution and its ability to discriminate electrons from hadrons, CALET enables a detailed search for various spectral structures in high-energy electron cosmic rays, with the capability to provide the first experimental evidence of the presence of a nearby astrophysical cosmic-ray source.

The electron spectrum analysis starts with detailed detector calibrations ranging from detector alignment to energy determination, followed by selection of well-reconstructed singly charged electron tracks, further quality cuts using shower concentration along the shower axis in the bottom layer of IMC, and analysis of the energy deposit distributions in the IMC and TASC layers and the shower shape difference between electromagnetic and hadronic showers. The geometric acceptance and selection efficiencies are calculated using the detailed Monte Carlo simulation of the CALET flight model, in which all of the relevant detector responses are taken into account. Background contamination due to the remaining protons is estimated and subtracted from the surviving events using simulated proton data, which reproduces the current observation results. Various consistency checks between data and MC simulation are performed using flight data.

A preliminary spectrum of total electrons (electrons + positrons) was obtained in an energy range of $10 \mathrm{GeV}$ to $1 \mathrm{TeV}$, based on a limited data sample of 18 months of observation. It will be published soon in a refereed journal.

35th International Cosmic Ray Conference - ICRC2017 -

10-20 July, 2017

Bexco, Busan, Korea

${ }^{*}$ Speaker. 


\section{Introduction}

High-energy cosmic-ray electrons provide an unique probe of nearby high-energy cosmic accelerators. Electrons rapidly lose energy via inverse Compton scattering and synchrotron emission during propagation in the galaxy. Since their diffusion distance over $1 \mathrm{TeV}$ is less than $1 \mathrm{kpc}$, there are a few potential $\mathrm{TeV}$ electron sources in the vicinity of the solar system. A precise measurement of the electron spectrum up to the $\mathrm{TeV}$ region might reveal interesting spectral features to provide the first experimental evidence of the presence of a nearby cosmicray source [1]. On the other hand, the prominent increase of the positron fraction over $10 \mathrm{GeV}$ has been established by PAMELA [2] and AMS-02 [3], which may require a primary source component for positrons in addition to the generally accepted secondary origin. Candidates for such primary sources range from astrophysical (pulsar) to exotic (dark matter) in nature. Since these primary sources emit electron-positron pairs, it is expected that the total electron spectrum exhibits a spectral feature, especially near the acceleration limit for the primary source.

The balloon experiments, ATIC [4] and PPB-BETS [5], measured the total electron spectrum up to $\mathrm{TeV}$ in relation to positron excess. Notably, ATIC has detected a prominent spectral feature at around $600 \mathrm{GeV}$, which could be related to dark matter annihilation [4]. The recent measurements in space with much higher statistics by AMS-02 [6] and Fermi/LAT [7], however, did not detect so far such a feature. Furthermore, the two precision measurements are not consistent with each other above $100 \mathrm{GeV}$. Despite a significant progress in the quality of the measurements in the past decade, there remains significant room to study the cosmic-ray electron spectrum, including the expected cutoff and the possible signature of a local accelerator in the $\mathrm{TeV}$ region. New measurements with a dedicated calorimetric instrument of sufficient thickness are critically important, where fine energy resolution is intrinsically guaranteed by total absorption of the primary particle energy. CALET [8] and DAMPE [9] are instruments of this kind currently operating in space. In the present paper, we report the first preliminary results of CALET electron flux from $10 \mathrm{GeV}$ to $1 \mathrm{TeV}$.

\section{CALET Instrumentation}

CALET docked at the Exposed Facility of the Japanese Experiment Module (JEM-EF) on the International Space Station (ISS) in August 2015 and has been collecting data [10] since October 2015. CALET has been designed for long-duration observations of high-energy cosmic rays onboard the ISS. The CALET calorimeter, shown in Fig. 1, has several unique and important characteristics. They include its ability to resolve in detail the initial development of showers, as well as the tracks generated by non-interacting minimum ionizing particles

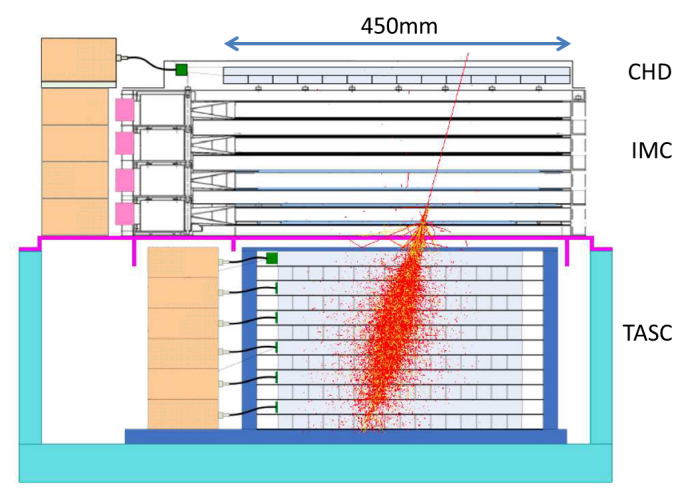

Figure 1: A cross-sectional view of the CALET detector with an example of a simulated $1-\mathrm{TeV}$ electron shower. (MIPs), and its capacity to precisely measure the energy of electrons in the $\mathrm{TeV}$ region as a result of its depth of 30 radiation-lengths $\left(X_{0}\right)$. These features are achieved through a combination of three primary detector sub-systems: particle identification and energy measurements are performed by the total absorption calorimeter (TASC), 
the $3 X_{0}$ thick imaging calorimeter (IMC) ensuring proper development of electromagnetic shower in its initial stage is used for track reconstruction, and charge identification is obtained from the charge detector (CHD). The key performance of each detector component was estimated based on a detailed Monte Carlo (MC) simulation [11] and was confirmed by several beam tests carried out primarily at the CERN-SPS beam-test facility [12, 13, 14].

A simulated 1-TeV electron shower is shown in Fig. 1. Given its thickness, the TASC perfectly contains TeV-energy electromagnetic showers. In contrast, the thickness of the instrument corresponds to a 1.3 interaction length for protons, and most of the secondary charged pions escape from the detector. This feature indicates a clear difference in shower shape, especially at the bottom part of TASC, resulting in the capability to identify electrons out of an otherwise overwhelming hadron background. Together with precision energy measurements, taking advantage of the total absorption of the electromagnetic shower, it is possible to derive an electron spectrum well into the $\mathrm{TeV}$ region with a simple and reliable analysis.

\section{Data Analysis}

We have analyzed flight data (FD) obtained by a high-energy shower trigger [10] for 536 days from October 13, 2015 to March 31, 2017. The total observational live time is 10,812 hours, and the live time fraction to total time is $84 \%$. Very stable and continuous observation has been accomplished during the data taking.

For a calorimeter like CALET, energy calibration is of the utmost importance. The method and associated uncertainties of energy calibration are described and summarized in Ref. [15]. Taking into account the detector responses, such as pedestal noise and several calibration uncertainties, detailed calibration achieved a fine energy resolution of $2 \%$ or better in the energy region from $20 \mathrm{GeV}$ to $20 \mathrm{TeV}$ ( $<3 \%$ for $10-20 \mathrm{GeV}$ ). Regarding temporal variations caused during the longterm observation, CALET is calibrated for each detector component by modeling the variations of MIP peak obtained from non-interacting particles (proton or helium) taken with a dedicated trigger mode. The gain change rate is less than $1 \%$ per month on average for all of the detector components except for a few low-gain channels in $\mathrm{CHD}$, and the variation rate decreases as time advances. The variation rate is less than $0.5 \%$ per month after one year from the start of operations. As another important calibration, the IMC fiber alignment is also calibrated using penetrating particles on orbit, which is very well-consistent with ground calibration results using atmospheric muons.

The Monte Carlo (MC) program is used to simulate physics processes and detector signals based on the simulation package EPICS [16] (EPICS9.20 / COSMOS8.00), which is updated by the accelerator beam test, with a detailed detector configuration. The MC event samples are generated in order to derive event selection and event reconstruction efficiencies, energy correction factor, and background contamination. These samples consist of down-going electrons and protons produced isotropically from the surface of a sphere with a radius of $78 \mathrm{~cm}$, which totally covers the instrument. With respect to detector response, electronics noise measured with pedestal distributions, photoelectron statistics obtained from MIP distributions, and crosstalk between fibers in the IMC are implemented in the MC, whereas the other items are taken into consideration through detector calibration.

Physics analysis, which consists of track and energy reconstruction, electron event selection, subtraction of proton contamination, and flux normalization, is performed using the CALET Level2 
(L2) data set [17] in which flight data (FD) and Monte Carlo simulation data (MC) are equivalent in format and in data quality.

Track reconstruction: Since some of calibrations and most of the selection parameters depend on the trajectory of an incoming particle, track reconstruction is one of the most important steps in data analysis. As a track reconstruction algorithm, in the present study, we adopt "electromagnetic shower tracking" (EM track) [13], which takes advantage of electromagnetic shower characteristics and of the IMC design concept. Thanks to appropriately arranged tungsten plates between IMC layers, shower developments for incoming electrons are clean and stable. By using the welldeveloped shower core at the bottom IMC layers as the origin of the track finding algorithm, very reliable and highly efficient track reconstruction becomes possible.

Event pre-selection: In order to minimize and accurately subtract proton contamination in electron identification, it is important to apply preselection of well-reconstructed and well-contained events having a charge of one. Furthermore, by removing events that are not included in MC samples, i.e., incidence from zenith angle greater than $90^{\circ}$ and heavier particles, equivalent event samples between FD and MC were obtained in order to be fed into the electron identification. This is the most important purpose of preselection. Preselection consists of offline trigger, the geometrical condition that the reconstructed track must traverse the instrument from CHD top to TASC bottom layer, track quality cut to ensure track reconstruction accuracy, charge selection using CHD, and proper shower-development requirements using likelihood and shower concentration at the IMC bottom layers. Combined efficiency of preselection for electrons is very high: $>90 \%$ above $30 \mathrm{GeV}$ to $3 \mathrm{TeV}, 85 \%$ at $20 \mathrm{GeV}$ and down to $60 \%$ at $10 \mathrm{GeV}$ due to trigger efficiency.

Energy reconstruction: In order to reconstruct the energy of primary electrons, an energy correction function is derived using the electron MC data after preselection. The energy deposit in the detector is obtained as the sum of TASC and IMC, where a simple sum is enough for TASC while compensation for energy deposits in tungsten plates is necessary for IMC. The correction function is then derived by calculating the average ratio of the true energy to the energy deposit sum in the detector. Owing to total absorption, the correction factor is very small, $\sim 5 \%$, up to the $\mathrm{TeV}$ region. Since the energy resolution is sufficient, there is no need to apply energy unfolding.

Electron identification: The last step of event selection is electron identification taking advantage of the shower shape difference between electromagnetic and hadronic showers. We applied two methods, i.e., simple two parameter cuts and multivariate analysis (MVA) based on machine learning, to understand systematic effects and stability of the resultant flux. Simple two parameter cut uses $K$ parameter defined by $K=\log _{10}\left(F_{E}\right)+R_{E} / 2 \mathrm{~cm}$, where $R_{E}$ is the second moment of lateral energy-deposit distribution in the TASC first layer with respect to the shower axis, and $F_{E}$ is the fractional energy deposit of TASC-Y6 layer sum with respect to the TASC total sum. For MVA analysis, we use the multivariate analysis toolkit TMVA [18] to train boosted decision trees (BDT) and to calculate BDT response. The discriminating variables are selected in an energy-dependent manner by using the variables with very good agreement between MC and FD. The discriminating variables above $500 \mathrm{GeV}$ are (1) $R_{E}$, (2) $F_{E}$, (3-6) parameters from the fit of longitudinal shower development in TASC, and (7-9) parameters to fit the pre-shower development with an exponential function in IMC. In order to maximize the rejection power against the abundant protons, especially at the highest energy region above $500 \mathrm{GeV}$, MVA has been adopted, while simple parameter cut was used below $500 \mathrm{GeV}$. 

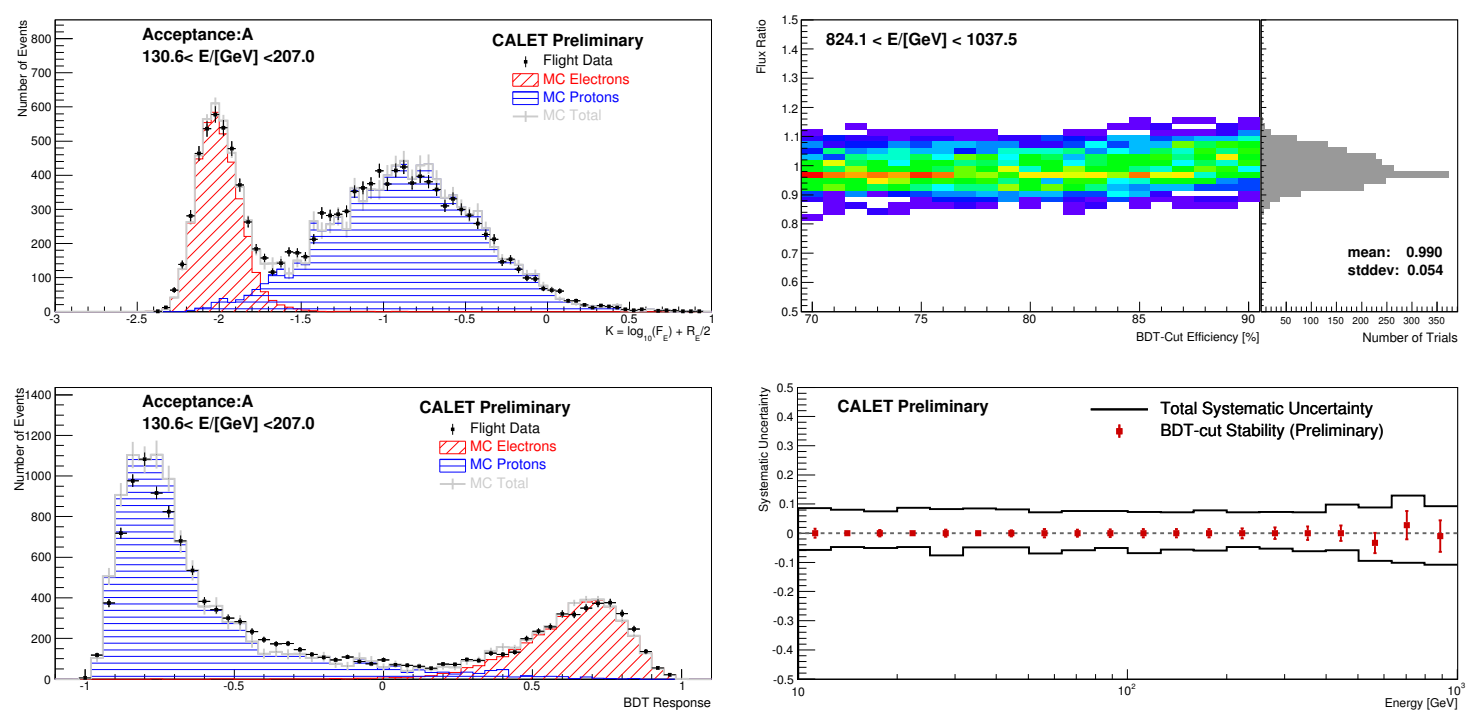

Figure 2: (Left) Examples of K-parameter (Top) and BDT response (Bottom) distributions in the $131<$ $E<207 \mathrm{GeV}$ bin. (Right Top) Stability of BDT analysis with respect to independent training samples and BDT-cut efficiency in the $824<E<1038 \mathrm{GeV}$ bin. Color maps show the flux ratio dependence on efficiency, where the bin value (number of trials) increases as color changes from violet, blue, green, yellow to red. Projections to Y-axis are shown as rotated histograms sharing Y-axes with color maps. (Right Bottom) Energy dependence of systematic uncertainties except for energy scale uncertainties in total and in electron identification using BDT. Note that center values below $500 \mathrm{GeV}$ are set to 0 where $\mathrm{K}$-cut is used for electron identification.

BDT analysis for e/p separation: In the BDT analysis, the whole set of MC data is equally separated into training and test samples. While training is carried out only using the training dataset, test samples are actually used to estimate the efficiency for electrons and contamination of protons. In order to take advantage of the distinctive shape of shower development for electrons, training was carried out separately for each geometry condition and for each finely binned energy region, with a bin-width factor of $10^{1 / 25}$. Examples of K-parameter and BDT response distributions are shown in the left-hand panels of Fig. 2, which demonstrate the separation power and very good agreements between FD and MC.

Subtraction of proton contamination: In order to extract the proton contamination in the final electron sample, template fittings of the K-parameter and BDT response were used, where normalization factors for $\mathrm{MC}$ electrons and $\mathrm{MC}$ protons are adopted as fitting parameters. The cut position corresponding to $80 \%$ efficiency is determined using the distribution of MC electrons. The contaminating protons are derived as the expected absolute number of events from the distribution of MC protons and the normalization factor, in order to subtract the background protons, regardless of the spectral shape of the electrons. The resultant contamination ratios of protons in the final electron candidates are $\lesssim 5 \%(\lesssim 8 \%)$ in the BDT (K-cut) analysis below $1 \mathrm{TeV}$. The contamination ratio will be much more reduced in further analysis.

Geomagnetic Cutoff Rigidity Measurements: Geomagnetic rigidity cutoff offers an universal energy scale to space based detectors [19]. To measure cutoff rigidity with CALET, we followed Fermi-LAT's recipe described in detail in Ref. [19, 7]. The left-hand panel of Fig. 3 shows one example of such measurements, where black and green squares show the low energy spectra taken at low rigidity cutoff region and high rigidity cutoff region $(1.00<L<1.14$ in Mcllwain $L)$, respectively. To derive the spectra, data taken with low-energy shower trigger $(E>1 \mathrm{GeV})$ are 
used instead of normal HE trigger. The secondary component was subtracted using azimuthal distribution. The extracted primary component was fitted by parameterization and compared with the calculated one (denoted as Tracer in the figure), which traces particle in earth's magnetic field (IGRF12) [20]. The difference between calculated and measured cutoff energy were taken as correction factor for absolute energy scale. The weighted average of the correction was found to be $1.035 \pm 0.009$ by combining three measurements carried out in $0.95<L<1.00,1.00<L<1.14$ and $1.14<L<1.25$, as shown in right-hand panel of Fig. 3. Since universal energy-scale calibration between different instruments is very important, we adopt the energy scale determined by rigidity cutoff to derive our spectrum.

\section{Systematic Uncertainties}

The main sources of systematic uncertainties are (i) energy scale, (ii) absolute normalization and (iii) electron identification.

(i) The energy scale determined by rigidity cutoff is $3.5 \pm 0.9$ (stat)\% higher than that by MIP calibration. While the reason for this discrepancy is not fully understood at present, it shows our level of understanding in absolute energy scale because two methods are totally independent. We therefore estimate systematic uncertainty of $3.5 \%$ in absolute energy scale. Since full dynamic calibration [15] is carried out relatively, it is reliable independent of absolute scale uncertainty.

(ii) The systematic uncertainty related to the absolute normalization arises from geometrical acceptance, live time measurement, long-term stability of the detector, track reconstruction, and various pre-selections. In contrast to magnet spectrometers, where $S \Omega$ varies as a function of the incident particle energy, $S \Omega$ is a pure geometrical factor for calorimeters and is to a good approximation energy independent. Since the geometry of the CALET detector is accurately measured on the ground and described in the MC model, the systematic errors due to the detector acceptance are small enough to be neglected. Other errors are taken into account by studying the stability of the spectrum for each contributing factor. For example, in order to estimate the systematics due to live time measurements, the stability of the spectrum with respect to the various dead time fractions is investigated. For tracking-related systematics, the dependence on the number of track hits and the difference between two independent tracking algorithms [21] are investigated.

(iii) To address the uncertainty in electron identification from protons, 100 sets of independent training were performed and the stability of the resultant flux was checked in each energy bin by changing the electron efficiency from $70 \%$ to $90 \%$ in $1 \%$ steps for the test sample corresponding to each training set. The results are shown in part in the Top Right panel of Fig.2. The reason the center value deviates from 1 is that the reference for the relative difference is not the average of all training sets, but rather one specific result used to derive the CALET electron spectrum. By combining all of the energy bins, the results are summarized in the Bottom Right panel of Fig. 2, where the average of all training samples with respect to the standard $80 \%$ case (specific training result) is shown by red squares, while error bars represent the standard deviation at each energy bin. The upper and lower edges of the error bars are taken as the systematic uncertainty taking into account the sign of the difference for each energy bin. Through the present study, we confirmed that our BDT analysis exhibits good stability with respect to training and cut efficiency.

Based on the above investigations, the systematic uncertainty bands that consider all of the components ranges from the live time dependence to electron identification except for the energy 

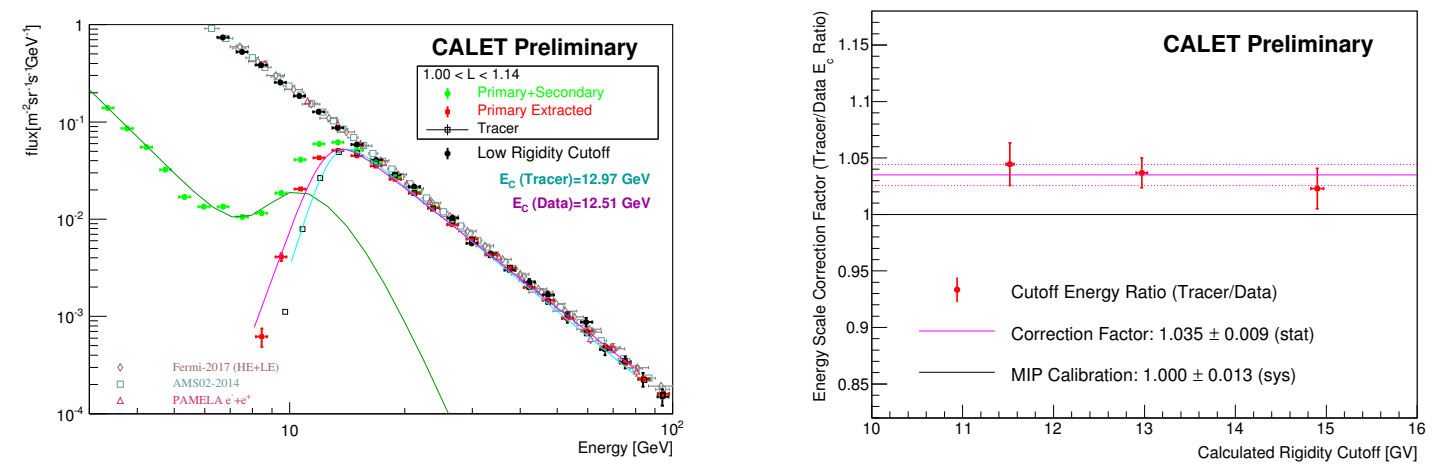

Figure 3: (Left) An example of cutoff rigidity measurement and comparison with calculation, (Right) Energy scale correction factors obtained from 3 different rigidity cutoff regions.

scale uncertainty are shown as black lines in the Bottom Right panel of Fig. 2, where each contribution is summed quadratically in order to estimate the total error. The various sources of systematic uncertainties have contributions at various energies. Among them, MC model dependence [22], comparison with simple two-parameter cut analysis in electron identification, BDT result stability, dependence on charge identification, tracking algorithm, and IMC shower concentration cut are important sources of systematic errors, while background statistics (MC proton statistics) is also important in the highest energy range. In the present study, we surveyed all of the viable choices in event selection, reconstruction and MC models, including those that are not optimal, and considered all of the differences in systematic uncertainty. Since, by design, CALET has intrinsic advantages in measuring the electron spectrum up to the $\mathrm{TeV}$ region, including (i) energy measurements with small correction by TASC and (ii) reliable tracking based on stable and clean shower development in IMC, all of the systematic uncertainties will be greatly reduced as our analysis proceeds further and as FD and MC statistics improve.

\section{Preliminary Results}

The differential flux between energy $E$ and $E+\Delta E[\mathrm{GeV}]$, with bin width $\Delta E[\mathrm{GeV}]$, is given by the following formula:

$$
\Phi(E)=\frac{N(E)-N_{\mathrm{BG}}(E)}{S \Omega \varepsilon(E) T(E) \Delta E(E)},
$$

where $\Phi(E)\left[\mathrm{m}^{-2} \mathrm{sr}^{-1} \mathrm{~s}^{-1} \mathrm{GeV}^{-1}\right]$ is the differential flux, $N(E)$ is the number of electron candidates in the corresponding bin, $N_{\mathrm{BG}}(E)$ is the number of background events estimated as MC protons, $S \Omega$ $\left[\mathrm{m}^{2} \mathrm{sr}\right]$ is the geometrical acceptance, $\varepsilon(E)$ is the detection efficiency for electrons, and $T(E)$ [s] is the observational live time. While $T(E)$ is basically constant, it is necessary to take into account the cutoff rigidity below $E<30 \mathrm{GeV}$, where the total live time is limited because we use only the data taken at the rigidity cutoffs below $6 \mathrm{GV}$. The electron plus positron spectrum measured with CALET will be published soon in a refereed journal.

Regarding the measurements in the $\mathrm{TeV}$ region, note that our constant-efficiency analysis is very important in order to avoid possible efficiency-related systematics. By taking advantage of the calorimeter thickness, it is possible for CALET to clearly discriminate electrons from protons in the $\mathrm{TeV}$ region, for example, as shown in Fig. 4. It is promising and also very important to further investigate the total electron spectrum with CALET by deepening the analysis and accumulating more statistics. 

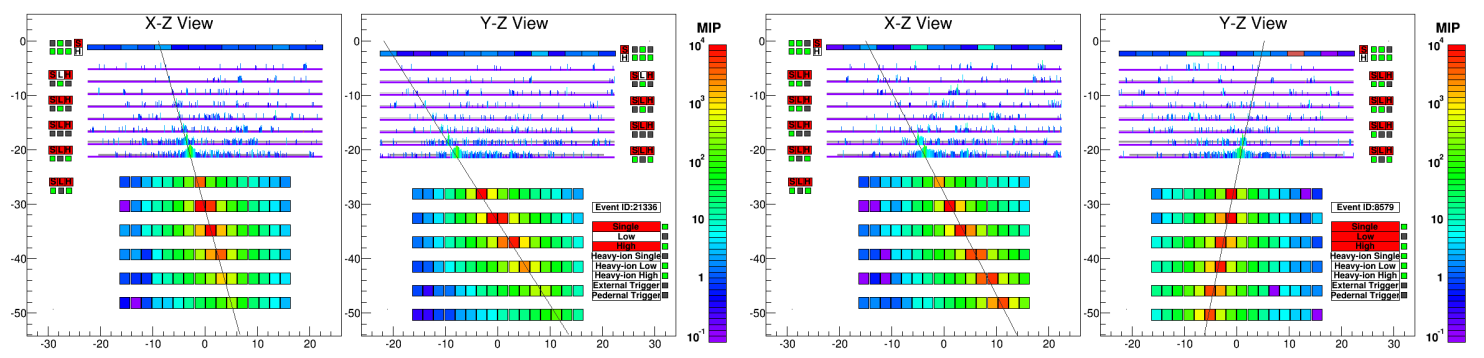

Figure 4: Examples of $\mathrm{TeV}$ event candidates showing energy deposit in each detector channel in the $\mathrm{X}-\mathrm{Z}$ and $\mathrm{Y}-\mathrm{Z}$ views. Left-hand panel shows an electron candidate (reconstructed energy of $3.05 \mathrm{TeV}$ ), and the right-hand panel shows a proton candidate (energy deposit sum of $2.89 \mathrm{TeV}$ ).

\section{Acknowledgements}

We gratefully acknowledge JAXA's contributions to the development of CALET and to the operations onboard the ISS. We also wish to express our sincere gratitude to ASI and NASA for their support of the CALET project. This work was supported in part by a JSPS Grant-in-Aid for Scientific Research (S) (no. 26220708) and by the MEXT-Supported Program for the Strategic Research Foundation at Private Universities (2011-2015) (No. S1101021) at Waseda University.

\section{References}

[1] T. Kobayashi et al., Astrophys. J. 601 (2004) 340; N. Kawanaka et al., Astrophys. J. 729 (2011) 93.

[2] O. Adriani et al., Nature 458 (2009) 607.

[3] L. Accardo et al., Phys. Rev. Lett. 113 (2014) 121101.

[4] J. Chang et al., Nature 456 (2008) 362.

[5] K. Yoshida et al., Adv. Space Res. 42 (2008) 1670.

[6] M. Aguilar et al., Phys. Rev. Lett. 113 (2014) 221102.

[7] A.A. Abdollahi et al., Phys. Rev. D. 95 (2017) 082007.

[8] S. Torii et al., Proc. of Science, Proc. of the 34th ICRC (The Hague, Netherlands), 1.

[9] J. Chang, Chinese Journal of Space Science 34 (2014) 550.

[10] Y. Asaoka et al., Proc. of Science, Proc. of the 34th ICRC (The Hague, Netherlands), 603.

[11] Y. Akaike et al., Proc. of the 32nd ICRC (Beijing, China), Vol.6, (2011) 371.

[12] M. Karube et al., Proc. of the 32nd ICRC (Beijing, China), Vol.6, (2011) 383.

[13] Y. Akaike et al., Proc. of the 33rd ICRC (Rio de Janeiro, Brazil), ID\#726 (2013).

[14] T. Niita et al., Adv. Space Res. 55 (2015) 2500.

[15] Y. Asaoka et al., Astropart. Phys. 91 (2017) 1.

[16] K. Kasahara, Proc. of 24th International Cosmic Ray Conference (Rome, Italy), Vol. 1 (1995) 399; "EPICS Home Page" http://cosmos.n.kanagawa-u.ac.jp/EPICSHome/.

[17] S. Ozawa et al., Proc. of the 35th ICRC (Busan, Korea), this conference (2017).

[18] A. Hocker et al., Proc. Sci., ACAT2007 (2007) 040 [arXiv:physics/0703039].

[19] M. Ackermann et al., Astropart. Phys. 35 (2012) 346.

[20] Erwan Thebault et al., Earth, Planets and Space 2015, 67.

[21] P. Maestro and N. Mori et al., Proc. of the 35th ICRC (Busan, Korea), PoS (ICRC2017) 208 (2017).

[22] L. Pacini et al., Proc. of the 35th ICRC (Busan, Korea), this conference (2017). 